\title{
Philosophiques
}

\section{Rescher, Nicholas. Essais sur les fondements de l'ontologie du procès, traduction et introduction par Michel Weber, Ontos-Verlag, 2006}

\section{Frédéric Tremblay}

Volume 34, numéro 2, automne 2007

URI : https://id.erudit.org/iderudit/017429ar

DOI : https://doi.org/10.7202/017429ar

Aller au sommaire du numéro

Éditeur(s)

Société de philosophie du Québec

ISSN

0316-2923 (imprimé)

1492-1391 (numérique)

Découvrir la revue

Citer ce compte rendu

Tremblay, F. (2007). Compte rendu de [Rescher, Nicholas. Essais sur les fondements de l'ontologie du procès, traduction et introduction par Michel Weber, Ontos-Verlag, 2006]. Philosophiques, 34(2), 419-421.

https://doi.org/10.7202/017429ar d'utilisation que vous pouvez consulter en ligne.

https://apropos.erudit.org/fr/usagers/politique-dutilisation/ 


\title{
Comptes rendus
}

\author{
Rescher, Nicholas. Essais sur les fondements de l'ontologie \\ $d u$ procès, traduction et introduction par Michel Weber, \\ Ontos-Verlag, 2006
}

Ce livre est la traduction d'une collection de textes tirés de quatre monographies de Rescher: Process Metaphysics, Process Philosophy, Nature and Understanding, et Inquiry Dynamics, regroupés sous trois thèmes, soit Prolégomènes, Métaphysiques du procès, et Épistémologie du procès. L'ensemble est précédé d'une brève préface du traducteur qui met en parallèle la philosophie du processus de Rescher avec celle de Whitehead. Dans les Prolégomènes, Rescher présente un relevé de l'histoire de la philosophie du processus avant d'en exposer les thèses générales. Il défend ces thèses contre les philosophies anti-processuelles, en particulier celle de P. F. Strawson, et indique quelques difficultés des ontologies substantialistes. Cette première partie forme donc la base des deux autres parties dans lesquelles Rescher applique l'interprétation processuelle à certaines sphères de la science, en commençant avec l'anthropologie philosophique et en proposant une analyse de la personne en termes (partiellement) processuels : une personne n'est pas une substance mais un agrégat de processus, dispositions, tendances, etc. Il l'applique ensuite aux sciences naturelles en s'attardant particulièrement au caractère kaléidoscopique de la complexité de la nature. Rescher s'intéresse en troisième lieu aux processus dans le contexte de la cognition, du développement scientifique et philosophique, et du réalisme métaphysique.

L'application de la perspective processuelle aux différentes sphères de la science n'a, en revanche, une valeur proprement scientifique que dans la mesure où l'ontologie processuelle est elle-même fondée. Attardons-nous donc maintenant sur la question des fondements de l'ontologie du processus qu'annonce le titre. Quels sont ces fondements ? Dans la première partie, une section est consacrée aux "Idées fondamentales » dans laquelle Rescher présente les deux thèses générales de la philosophie du processus : 1 ) "les choses nécessitent les procès"; et 2) "les procès sont plus fondamentaux que les choses " (p. 44). La première thèse est causale et veut que les processus soient causalement premiers aux choses, la seconde concerne la constitution ontique des choses et veut que ces dernières soient ultimement constituées de processus. Les deux thèses reposent implicitement sur une troisième suivant laquelle il existe des processus sans propriétaires ou, pour suivre la traduction de Weber, "procès impropres ".

Un processus avec propriétaire (owned process) est le processus d'une substance ou, du moins, un processus dont l'existence dépend d'une substance. Un processus sans propriétaire (unowned process), au contraire, est un processus sans support substantiel, free floating pour employer l'expression de Rescher. Ainsi, le processus sans propriétaire est séparable de toute substance et peut exister indépendamment d'elle. Rescher exprime cette idée de la façon suivante: «Du point de vue du philosophe du procès, l'existence de procès impropres est particulièrement importante, car elle montre que le domaine du procès est complémentaire et séparable du domaine des choses substantielles » (p. 56).

La thèse de l'existence de processus impropres (ou séparabilité du processus) est une condition nécessaire aux deux thèses exposées plus haut. En effet, la thèse suivant laquelle les processus sont causalement premiers aux substances implique qu'il y ait un 
processus séparé d'une substance qui soit causalement première à la substance. Et, ensuite, la thèse suivant laquelle les processus sont plus fondamentaux que les substances présuppose que les substances sont, au niveau microscopique, ultimement constituées de processus sans propriétaires (je présume que Rescher les conçoit comme étant impropres, car autrement il n'y aurait pas lieu de parler d'une relation de fondation).

Mais cette thèse ne tient pas compte de la corrélation à chaque niveau de granularité entre les mouvements et leurs substances correspondantes: les galaxies sont constituées non pas de rotations impropres mais de systèmes stellaires en rotation, euxmêmes constitués d'amas de corps célestes en rotation comme notre système solaire. Plus bas dans le spectre des niveaux de granularité, les molécules sont constituées d'atomes, lesquels forment une structure relativement stable moyennant des processus d'attraction et de répulsion, les atomes sont à leurs tours constitués d'électrons en interaction avec le nucleus, dont les parties (protons et neutrons) sont mues par des processus d'attraction réciproque. Ces dernières sont constituées de trois quarks en interaction, etc.

Ainsi, la dichotomie et la correspondance entre substance et processus semblent continues et nécessaires aussi loin qu'il nous est permis de descendre ou de monter dans l'échelle des niveaux de granularité, de sorte que nous n'observons aucun processus réel qui n'est pas corrélé à sa substance, et vice-versa, aucune substance qui n'est pas en pair avec son processus corrélatif propre. En somme, il apparaît invraisemblable de prime abord qu'il existe des processus impropres ou séparés. Et sans une justification de cette thèse invraisemblable, le projet de la philosophie du processus demeure dénué de fondement.

Or, Rescher ne démontre nulle part la validité de cette thèse. Ou peut-être croit-il l'avoir confirmée de façon inductive par les exemples de processus impropres qu'il propose. Ces exemples sont " la baisse de la température, le changement du climat, le flash de l'éclair, la fluctuation d'un champ magnétique» (p. 55). Si ces processus existaient réellement à l'état séparé (c.-à-d., sans propriétaire), alors Rescher pourrait s'appuyer sur des évidences empiriques. Aucun des exemples, cependant, n'est convaincant; chacun d'eux nous semble réductible à un processus propre (c.-à-d. avec propriétaire). Examinons les exemples un à un.

Réchauffements et refroidissements se produisent toujours dans une portion déterminée d'air ou d'eau (ou autre matière) spatialement localisable, et cette portion déterminée est précisément la substance correspondant au processus. La température est également définie, à un niveau plus raffiné de granularité, comme l'énergie cinétique de particules dans un certain système. Suivant cette définition, le processus de baisse de la température est un processus de diminution de l'énergie cinétique des particules, et le réchauffement une augmentation de celle-ci. L'exemple du changement de climat reçoit la même explication à une plus grande échelle.

Prenons maintenant l'exemple du flash de l'éclair. La traduction de lightning par éclair ne rend pas l'effet recherché, car lightning est un terme de masse, alors qu'éclair est un terme singulier. Rescher semble inférer qu'aux termes de masses correspondent une masse indéterminée plutôt qu'une substance et qu'en conséquence le processus du flash de l'éclair n'a pas de propriétaire. Mais si certains processus correspondaient à de telles masses, ils ne pourraient pas êtres dits impropres, car ils auraient des masses comme propriétaires. Il faudrait déjà pour cela que Rescher ait défendu l'existence de masses indéterminées, ce qu'il n'a pas fait (du moins pas dans cette collection de textes).

L'exemple de la fluctuation d'un champ magnétique est plus difficile à discuter, car il présuppose une certaine compréhension des équations de Maxwell ou de la 
mécanique quantique, mais il est certain qu'il n'y a pas de champ magnétique sans dipôle magnétique, et pas de dipôle magnétique sans un support quelconque (aimant, courant électrique, corps céleste, etc.), de même qu'il n'y a pas de champ magnétique actuel sans électrons qui se meuvent d'un pôle à l'autre. En somme, on n'observe point de champ magnétique sans support.

Plus loin dans le même chapitre, Rescher présente deux nouveaux exemples: la chute de la demande économique et l'érosion de la ligne côtière. Or, de la même façon, la chute de la demande économique est une statistique induite à partir d'un nombre déterminé d'individus, et l'érosion de la ligne côtière est toujours constituée d'un nombre déterminé de processus spécifiques de dissociation, ayant chacun pour support un nombre déterminé de grains de sable (ou autre), spatiotemporellement localisables.

Ainsi, parce que les thèses générales de la philosophie du processus reposent sur la thèse de la séparabilité de certains processus et que Rescher n'a pas démontré que ceuxci existent effectivement de façon séparée, alors la philosophie (rescherienne) du processus ne repose sur aucun fondement satisfaisant, du moins dans sa version ontologique, dite "forte» (et dans la mesure où la version épistémologique, dite "faible ", reposerait sur les mêmes fondements, alors elle serait tout aussi problématique). En effet, sans la thèse de l'existence de processus impropres, celles de la primauté causale et du caractère fondamental des processus se dissolvent, car pour être premier ou fondamental par rapport à autre chose il faut d'abord que l'un puisse se trouver sans l'autre.

La traduction est généralement conforme. Toutefois la reddition de owned et unowned par «propre» et «impropre» est malheureusement cryptique. De plus, le choix surprenant de "procès " plutôt que "processus » n'est nulle part sérieusement motivé. Weber se contente d'une note dans le lexique où il écrit que process est "parfois traduit, pour des raisons euphoniques, [par] "processus" » (p. 276). Or "processus » est préférable pour des raisons plus sérieuses que la simple euphonie. Parmi ces raisons, notons d'abord que le premier sens de "procès" est juridique, de sorte que l'expression « ontologie du procès » pourrait être méprise pour une espèce de l'ontologie du droit et, ensuite, que l'usage déjà établi du terme processus dans le vocabulaire francophone de la philosophie n'a rien de problématique et qu'il est donc superficiel de lui chercher un substitut.

FRÉDÉRIC TREMBLAY

SUNY at Buffalo et IFOMIS (Saarbrücken)

\section{Zeimbekis, John. Qu'est-ce qu'un jugement esthétique? Vrin, coll. Chemins philosophiques, 2006, $128 \mathrm{p}$.}

Cette nouvelle parution relève le défi de présenter une notion incontournable qui a alimenté les querelles et débats en esthétique au dix-huitième siècle. En effet, la notion de jugement esthétique, conçue à l'époque en tant qu'affirmation portant sur la beauté d'un objet ( «x est beau », "y est élégant », etc.) a alimenté des querelles épistémologiques sur la nature et le statut de tels jugements. Par exemple : à quelles conditions puis-je dire, si j'affirme que " $\mathrm{x}$ est beau », que mon affirmation est vraie? On l'aura deviné : l'enjeu est de déterminer à quelles conditions ce type d'affirmation peut avoir un caractère objectif et par conséquent à quelles conditions on pourrait en faire la pierre d'assise d'un discours sur le beau et sur la notion de plaisir. Le traitement 